\title{
Critical analysis of antibacterial agents in clinical development
}

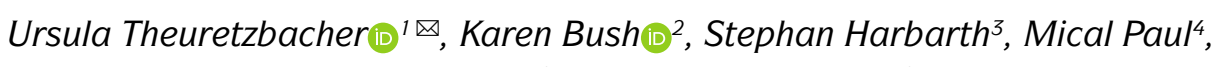
John H. Rex@5 ${ }^{5}$, Evelina Tacconelli ${ }^{6}$ and Guy E. Thwaites ${ }^{7,8}$

Abstract | The antibacterial agents currently in clinical development are predominantly derivatives of well-established antibiotic classes and were selected to address the class-specific resistance mechanisms and determinants that were known at the time of their discovery. Many of these agents aim to target the antibiotic-resistant priority pathogens listed by the WHO, including Gram-negative bacteria in the critical priority category, such as carbapenem-resistant Acinetobacter, Pseudomonas and Enterobacterales. Although some current compounds in the pipeline have exhibited increased susceptibility rates in surveillance studies that depend on geography, pre-existing cross-resistance both within and across antibacterial classes limits the activity of many of the new agents against the most extensively drug-resistant (XDR) and pan-drug-resistant (PDR) Gram-negative pathogens. In particular, cross-resistance to unrelated classes may occur by co-selection of resistant strains, thus leading to the rapid emergence and subsequent spread of resistance. There is a continued need for innovation and new-class antibacterial agents in order to provide effective therapeutic options against infections specifically caused by XDR and PDR Gram-negative bacteria.

There is a widely acknowledged need for new antibacterial agents to address the global increase in resistance, and this need for new agents is especially urgent for the treatment of antibiotic-resistant Gram-negative bacteria. In early 2017, the WHO convened a group of experts that used a multi-criteria decision analysis method to prioritize the need for new drugs to treat antibiotic-resistant bacteria ${ }^{1}$. The WHO assigned the highest priority to antibacterial drug research and development for the Gram-negative bacteria Acinetobacter, Pseudomonas and species of Enterobacterales that are resistant to carbapenems and are usually extensively drug resistant (XDR) ${ }^{1}$. The same year, the WHO released a clinical pipeline report, which was updated in 2018 and 2019 (REFS ${ }^{2,3}$; WHO clinical pipeline report). The clinical pipeline reports analysed antibiotics and biologics according to their activity against the criticalpriority pathogens carbapenem-resistant Acinetobacter baumannii (CRAB), carbapenem-resistant Pseudomonas aeruginosa (CRPA), extended-spectrum $\beta$-lactamase (ESBL)-producing Enterobacterales and carbapenemresistant Enterobacterales (CRE). The level of innovation in the global clinical pipeline was assessed on the basis of the absence of pre-existing cross-resistance to currently used antibacterial drugs ${ }^{4}$.

In this Review, we summarize the current published literature and the publicly available information on antibacterial agents in all phases of clinical development, according to the WHO pipeline report ${ }^{3}$. This Review is limited to antibacterial agents that were in clinical development for systemic human use and that did not yet have regulatory approval anywhere in the world for human use. Additionally, drugs against Clostridioides difficile infection are included, although mostly these agents are not absorbed systemically, because of their oral administration. The principal focus is on the ability of new agents to treat infections caused by bacteria that are XDR or pan-drug-resistant (PDR), the main driver of research and development ${ }^{5}$. Thus, bacteriological information is an important basis for this analysis. We further analyse the gaps in the global clinical pipeline and the need for future antibacterial agents. Although other therapeutic and preventive approaches have been developed, in this Review we focus on direct-acting small molecules for therapeutic purposes ('traditional antibiotics'). Non-traditional therapies ${ }^{6,7}$ and anti-tuberculosis treatments ${ }^{8,9}$ have been extensively reviewed elsewhere. Discussing the economic challenges of antibacterial drug development is beyond the scope of this Review.

\section{Top three resistant pathogens}

Carbapenem-resistant Acinetobacter baumannii. The prevalence of carbapenem resistance among Acinetobacter spp. is extremely variable, ranging from $<10 \%$ to $>90 \%\left(\right.$ REF $\left.^{10}\right)$. XDR is common because of the diverse and extensive arsenal of chromosomally encoded 
$\beta$-lactamase

$\beta$-lactamases are a

heterogeneous group of

enzymes produced by

bacteria, used to hydrolyse

$\beta$-lactam antibiotics.

Porin

Proteins that form nonspecific channels allowing the transport of molecules across the outer membranes of Gram-negative bacteria.

Penicillin-binding protein Bacterial enzymes involved in bacterial cell wall biosynthesis that are the target of penicillin and all the other antibiotics of the $\beta$-lactam class. and acquired resistance genes carried by this pathogen (FIG. 1). Overexpression of the intrinsic chromosomally encoded $\beta$-lactamase ADC (class $C \beta$-lactamase in A. baumannii) and of OXA-51-like enzymes, as well as of diverse acquired $\beta$-lactamases - such as OXA-23-like, OXA-24/40-like, OXA-58-like and class A $\beta$-lactamases (for example, TEM and SHV) - found on a range of mobile genetic elements, is the main resistance mechanism against $\beta$-lactam antibiotics ${ }^{11}$. Combinations of different $\beta$-lactamases and the accumulation of other resistance determinants, such as aminoglycosidemodifying enzymes, porin deficiencies, efflux or target protein modifications, render CRAB resistant to most of the usually available antibacterial drugs and increasingly also to tigecycline and colistin. XDR and PDR isolates are routinely being reported in some countries ${ }^{12,13}$.

Carbapenem-resistant Pseudomonas aeruginosa. CRPA is genetically diverse and usually exhibits more than one resistance mechanism, including porin deficiency (OprD), hyperproduction of the chromosomally encoded cephalosporinase AmpC, efflux pumps and various class-specific resistance mechanisms (for example, mutations in quinolone resistance-determining regions $)^{14}$. These chromosomally encoded resistance determinants, alone or in combination, affect carbapenems and other $\beta$-lactams differently, and diverse combinations are prevalent in isolates from distinct countries $^{14}$. Loss of porins is the most common mechanism leading to carbapenem resistance. Overexpression of one of the several efflux pumps (for example, mexB or mexY) is a nonspecific mechanism that especially affects cefepime, aminoglycosides, fluoroquinolones and meropenem ${ }^{15}$. Acquired resistance mechanisms such as $\beta$-lactamases (for example, ESBLs or class B metallo$\beta$-lactamases (MBLs)) are often co-transferred with genes that encode aminoglycoside-modifying enzymes, but they are found less frequently than resistance conferred by chromosomally encoded genes. The prevalence of MBLs, especially VIM and IMP, is highly variable in different countries, and they are becoming increasingly widespread in some regions ${ }^{14}$. The accumulation of several resistance mechanisms to different antibacterial classes can lead to XDR or even PDR strains ${ }^{16}$ (FIG. 1).

Carbapenem-resistant Enterobacterales. Carbapenem resistance in Enterobacterales (mainly Klebsiella pneumoniae, but also Enterobacter cloacae, Escherichia coli,

\footnotetext{
Author addresses

${ }^{1}$ Center for Anti-Infective Agents, Vienna, Austria.

2Department of Biology, Indiana University, Bloomington, IN, USA.

${ }^{3}$ Infection Control Program, World Health Organization Collaborating Centre on Patient Safety, Geneva University Hospitals and Faculty of Medicine, Geneva, Switzerland. ${ }^{4}$ Diseases Institute, Rambam Health Care Campus. Ruth and Bruce Rappaport Faculty of Medicine, Technion - Israel Institute of Technology, Haifa, Israel.

${ }^{5}$ F2G Ltd., Wellesley Hills, MA,, USA.

${ }^{6}$ Infectious Diseases, Department of Diagnostic and Public Health, Verona University Hospital, Verona, Italy.

${ }^{7}$ Oxford University Clinical Research Unit, Ho Chi Minh City, Vietnam.

${ }^{8}$ Centre for Tropical Medicine and Global Health, Nuffield Department of Medicine, University of Oxford, Oxford, UK.
}

Citrobacter spp. and Serratia marcescens) is caused by the production and high diversity of all $\beta$-lactamase classes (class A, class B, class $\mathrm{C}$ and class $\mathrm{D}$ ), which are often present in combination and confer resistance to almost all $\beta$-lactam antibiotics (FIG. 1). The epidemiology of $\beta$-lactamases is complex, as exemplified by the production of MBLs (often NDM type), which ranges from $1 \%$ in the United States to $100 \%$ in the Asia-Pacific region $^{17,18}$, with prevalences of NDM-producing E. coli of $83 \%, 13 \%, 1 \%, 1 \%$ and $2 \%$ in Asia, Europe, America, Africa and Oceania, respectively ${ }^{19}$. By contrast, the KPC class A carbapenemases are more widely distributed in the Americas, Korea and China ${ }^{20}$. In Europe, the prevalence of carbapenem resistance also differs greatly, with the highest rates in Mediterranean and Balkan countries $^{21}$. Even the inter-hospital prevalence of CRE and individual resistance mechanisms can range from $<10 \%$ to $90 \%$. New $\beta$-lactamase variants with a range of amino acid substitutions, increased hydrolysis of the $\beta$-lactam antibiotic, or amplification of the bla gene are commonly described. In addition, a diversity of KPC-2 and KPC-3 variants conferring resistance to ceftazidime-avibactam are circulating in the United States and Europe ${ }^{22-24}$. The production of $\beta$-lactamases may be accompanied by other resistance mechanisms - such as porin deficiencies with a similar, highly diverse epidemiology, upregulated efflux pumps in E. coli and, rarely, insertions into E. coli penicillin-binding protein 3 (PBP3) - that affect the microbiological activity of cephalosporins and monobac$\operatorname{tams}^{25,26}$. All these resistance-mechanism-related factors explain the high variability of susceptibility rates to new $\beta$-lactam-based therapies against CRE. In addition, CRE are also often resistant to unrelated antibacterial classes, such as fluoroquinolones, aminoglycosides, fosfomycin and tigecycline, thus making them XDR and sometimes $\mathrm{PDR}^{27}$. Associated resistance to colistin and tigecycline is linked to worse clinical outcomes ${ }^{28,29}$. In Greece, prevalences of $\sim 30 \%$ for XDR and $~ 5 \%$ for PDR have been reported ${ }^{27}$. The propensity for specific clones of K. pneumoniae to spread in hospital environments has been shown to correlate with the degree of resistance; distressingly, carbapenemase-producing isolates have the highest transmissibility ${ }^{30,31}$.

\section{$\beta$-lactam-based clinical pipeline}

As is shown by the four recently approved $\beta$-lactamase inhibitor (BLI) combinations (two approved since 2017) (TABLE 1), the clinical pipeline (TABLE 2) is characterized by new derivatives of the $\beta$-lactam class or the functional class of BLIs, most of them focused on improvements in the coverage of Enterobacterales ${ }^{3}$. The renaissance of $\beta$-lactam antibiotics in the beginning of the 21 st century has enriched the therapy options and addressed class-specific resistance due to the production of $\beta$-lactamases by either combining a $\beta$-lactam-containing molecule with a BLI or modifying it to prevent hydrolysis.

$\beta$-lactamase inhibitors and new combinations. BLIs have seen a remarkable evolution from $\beta$-lactam-derived molecules to new chemical classes, the diazabicyclooctanes (DBOs) and boronate classes ${ }^{32}$. The DBO class has evolved from mostly functional BLIs, with avibactam-ceftazidime 
CRAB

OXA-23, OXA-24/40, OXA-58-like TEM, SHV, CTX-M, MBLs

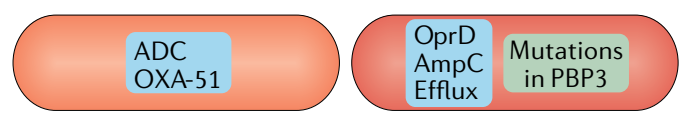

Fig. 1 Relevance of resistance determinants. The resistance determinants in carbapenem-resistant Acinetobacter baumannii (CRAB), carbapenem-resistant Pseudomonas aeruginosa (CRPA) and carbapenem-resistant Enterobacterales (CRE) are diverse and mostly associated with nonspecific or acquired resistance to other, unrelated antibiotic classes. Carbapenem resistance is frequently caused by the interplay between common (blue) and less common and variable resistance determinants (green). Chromosomally encoded resistance determinants are depicted inside a cell shape, while acquired ones are shown outside the shape. In CRAB, the common resistance determinants are intrinsic chromosomally encoded $\beta$-lactamases and acquired enzymes that are usually not inhibited by $\beta$-lactamase inhibitors (BLIs). CRPA strains are characterized by chromosomally encoded resistance determinants (OprD deficiency, overexpression of efflux pumps and $\mathrm{AmpC}$ ). Mutations in penicillin-binding protein 3 (PBP3) are still rare and affect the combination partner of BLIs. In extensively drug-resistant and pan-drug-resistant strains, additional, horizontally transferred resistance may accumulate. CRE are dominated by $\beta$-lactamases of all classes with high geographical variation. Porin deficiency may be combined with $\beta$-lactamases. In some areas, carbapenem resistance is associated with resistance to last-resort antibiotic options. MBL, metallo- $\beta$-lactamases; ESBL, extended-spectrum $\beta$-lactamase.

Minimal inhibitory

concentrations

(MICS). The lowest

concentrations of different

antibacterial compounds

that prevent visible growth

of bacteria.

Non-fermenters

A heterogeneous group of

bacteria that cannot use glucose and that thus are unable to generate energy through the fermentation of glucose. Important bacteria of this group are Pseudomonas and Acinetobacter

Clinical breakpoint

The chosen concentration of an antibiotic that defines whether a species of bacteria is susceptible or resistant to the antibiotic, which is used to predict the clinical outcome. as the first DBO combination, to compounds with additional relevant intrinsic antibacterial activity, through binding to PBP2 in enteric bacteria, one of the target proteins of $\beta$-lactam antibiotics (TABLE 3). This intrinsic antibacterial activity, albeit modest in some cases, contributes to a synergistic effect that may even extend the spectrum of the combination to some strains with $\mathrm{MBL}$ production, even if the MBLs are not inhibited. Boronate BLIs lack an intrinsic effect but have evolved into BLIs with a wider inhibitory spectrum to include some MBLs such as NDM and VIM (taniborbactam and the preclinical BLI QPX7728). Given that $\beta$-lactamases are mechanistically and structurally distinct, finding a BLI that is equally effective against all classes and relevant enzymes remains difficult ${ }^{33,34}$.

The BLIs vaborbactam (boronate) and relebactam (DBO) have been approved recently in combination with meropenem and imipenem, respectively (TABLE 1). Vaborbactam inhibits class A and class $C \beta$-lactamases, whereas meropenem is not hydrolysed by class $C$ enzymes $^{35}$. Relebactam is a DBO with inhibitory activity similar to that of avibactam against $\mathrm{KPC}^{36}$. Resistance against relebactam-imipenem in K. pneumoniae is mainly caused by MBLs, OXA-48, or GES carbapenemases or overexpression of the gene encoding KPC. The susceptibility rates in imipenem-non-susceptible K. pneumoniae and P. aeruginosa are $50 \%$ to $80 \%$ (REF. ${ }^{37}$ ). Thus, considerable pre-existing cross-resistance has been documented. BLIs cross the outer membrane of K. pneumoniae using OmpK35 and, preferably, OmpK36 $\left(\mathrm{REFS}^{35,38}\right)$. In the case of concomitant porin deficiencies (an as-yet-rare mechanism), the BLI combinations would be ineffective.

Currently, nine BLI combinations (one $\beta$-lactam, six DBOs and two boronates) are in clinical development. A tenth boronate BLI is ready for phase I but is not included yet in the clinical pipeline (TABLE 3). Aztreonam-avibactam is shown in TABLE 3 but not in TABLE 2, because it is a combination of registered components. This combination unites the activity of aztreonam against MBL-producing bacteria and the inhibitory activity of avibactam against class $\mathrm{A}$, class $\mathrm{C}$ and some class $\mathrm{D}$ (OXA) enzymes, thus covering a broad spectrum of $\beta$-lactamases. Other than belonging to different chemical classes (but the same functional class), the BLIs differ in the extents to which they affect bacteria directly, owing to PBP2 binding (DBOs) and an extended inhibitory coverage that in some compounds includes at least some MBLs (taniborbactam and QPX7728 in preclinical development).

The most advanced DBO in clinical development is durlobactam (ETX2514; intravenous only) with inhibitory activity against class $A$ and class $C \beta$-lactamases and a broad range of class $\mathrm{D} \beta$-lactamases beyond OXA- 48 (for example, the OXA-23, OXA-24/40, OXA-51 and OXA-58 families). Because these $\beta$-lactamases are the most prevalent resistance determinants in CRAB, the relevant company decided to develop this BLI in combination with sulbactam ( $\beta$-lactam-based BLI and PBP3 inhibitor) for the treatment of $A$. baumannii infections. Resistance to the combination is currently rare and is driven by the presence of MBLs (such as NDM-1) or mutations in PBP3, which is the target of sulbactam ${ }^{39}$. In countries with extremely high resistance prevalence in Acinetobacter species, such as India, resistance to durlobactam-sulbactam is due to NDM-producing isolates $^{40}$. On the basis of whole-genome-sequencing analyses, changes in the expression of efflux pumps may additionally reduce susceptibility ${ }^{41}$.

Nacubactam and zidebactam are DBOs with inhibitory activity against class $A$, class $C$ and some class D $\beta$-lactamases, and they bind to $\mathrm{PBP} 2$, thus showing synergistic activity in Enterobacterales ${ }^{33}$. This effect arises when the PBP2-directed activity of nacubactam is combined with PBP3-targeted agents such as cefepime $^{42}$, whereas meropenem primarily binds to PBP2 in E. coli. Nacubactam, at lower minimal inhibitory concentrations (MICs) ( $\leq 4 \mathrm{mg} / \mathrm{l}$ if tested alone), dominates the combination activity through PBP2 inhibition and may contribute to activity against MBL producers at high nacubactam concentrations ${ }^{43}$. At higher MICs (MIC > $4 \mathrm{mg} / \mathrm{l}$ ), nacubactam contributes to the combination activity against bacteria with class $\mathrm{A}$ or class $C \beta$-lactamases, contingent on $\beta$-lactamase inhibition $^{42}$. Nacubactam has no intrinsic effect on non-fermenters ${ }^{42,44}$. Similarly, zidebactam binds to PBP2 and has intrinsic activity against E. coli and Klebsiella spp., but insufficient activity against Enterobacter spp. and no activity against Pseudomonas and Acinetobacter spp. ${ }^{45}$. In combination with cefepime (which inhibits PBP3), zidebactam adds these PBP2-inhibiting potencies in E. coli and Klebsiella spp. As expected, the activity against CRPA is mediocre or insufficient (close to or above the clinical breakpoint for cefepime in $50 \%$ of the strains, and absent in $\mathrm{CRAB}^{45,46}$ ). Reduced susceptibility to cefepime-zidebactam is mostly associated with MBLs (IMP or VIM) or with combinations of mechanisms such as the overexpression of MexAB-OprM or MexXY efflux pumps, diminished OprD function and high-level AmpC production ${ }^{46}$. 
Taniborbactam is a bicyclic boronate and acts as a dual-action inhibitor of both serine- $\beta$-lactamases (SBLs) and MBLs ${ }^{47,48}$. The broad inhibitory activity includes class $A$ and class $C$ enzymes as well as the MBLs NDM and VIM, but not IMP, and moderately the OXA-48 carbapenemase (class D) ${ }^{47}$. In combination with cefepime, the MICs against NDM-producing and VIM-producing Enterobacterales are increased, and $10 \%$ to $20 \%$ of strains are resistant ${ }^{49}$. Given the scant knowledge of boronate-modifying enzymes, it will be of particular interest to observe whether unprecedented drug-modifying reactions emerge in response to clinical use $^{32}$. Cefepime-non-susceptible or meropenem-nonsusceptible $P$. aeruginosa show $20 \%$ to $30 \%$ resistance to taniborbactam-cefepime and thus can only be used for confirmed susceptible strains $s^{50}$. Another similar boronate, QPX7728, is in preclinical studies. Although the latter shows an inhibitory spectrum similar to that of taniborbactam ${ }^{51}$, the main difference is its potential for intravenous and oral formulations.

Three BLI combinations for oral use are in clinical development, thus far focused mainly on development for treating urinary tract infections (UTIs): one boronate BLI and two DBOs. The boronate BLI VNRX-7145 (prodrug, active compound VNRX-5236) is combined with the oral cephalosporin ceftibuten. Its spectrum encompasses ESBL-producing, KPC-producing and OXA-48-producing Enterobacterales ${ }^{52}$. Some weakness in the enzyme inhibitory activity is seen with selected OXA and class C enzymes ${ }^{53}$. VNRX-7145 restores the susceptibility of ceftibuten-non-susceptible Enterobacterales in $87 \%$ of strains, most efficiently in ESBL or KPC producers ${ }^{54}$. The oral DBO ETX0282 (prodrug, active compound ETX1317) is combined with the oral cephalosporin cefpodoxime proxetil (prodrug) and is active against Enterobacterales strains producing ESBL, KPC or OXA-48-like $\beta$-lactamases. ETX0282 has intrinsic activity through PBP2 binding, with useful activity against Enterobacterales and synergy depending on its concentration. Whether this synergistic effect will be sufficient for treating MBL-producing strains remains to be seen ${ }^{55}$. The second oral DBO is ARX-1796, an oral prodrug of avibactam that has mostly insufficient PBP2 inhibitory activity ${ }^{56}$.

Finally, the penicillanic acid sulfone BLI enmetazobactam is similar to tazobactam, with improved inhibitory activity of ESBL and OXA-48 enzymes in Enterobacterales, and is being developed in combination with an optimized dosage regimen of cefepime $e^{57}$. Cefepime is easier to potentiate than piperacillin, therefore achieving lower MICs of the combination in wild-type strains as well as in ESBL, and some KPC-2

Table 1 | Antibacterial agents that have been approved since 2017 by the FDA or EMA (modified according to the WHO report ${ }^{3}$ )

\begin{tabular}{|c|c|c|c|c|c|c|c|c|c|c|c|c|}
\hline \multirow[t]{2}{*}{$\begin{array}{l}\text { Name } \\
\text { (trade name) }\end{array}$} & \multirow[t]{2}{*}{$\begin{array}{l}\text { Approved by } \\
\text { (date, indication) }\end{array}$} & \multirow[t]{2}{*}{ Antibiotic class } & \multirow{2}{*}{$\begin{array}{l}\text { Route of } \\
\text { administration } \\
\text { (market } \\
\text { authorization holder) }\end{array}$} & \multirow[t]{2}{*}{ Indications } & \multicolumn{4}{|c|}{$\begin{array}{l}\text { Expected } \\
\text { activity against }\end{array}$} & \multicolumn{4}{|c|}{ Characteristics } \\
\hline & & & & & 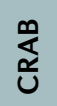 & $\frac{\nwarrow}{\frac{\nwarrow}{0}}$ & $\underset{\sim}{\stackrel{\sim}{u}}$ & 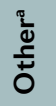 & 农 & $\dot{u}$ & $\vdash$ & 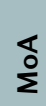 \\
\hline \multirow{2}{*}{$\begin{array}{l}\text { Delafloxacin } \\
\text { (USA: Baxdela, EU: } \\
\text { Quofenix) }\end{array}$} & $\begin{array}{l}\text { FDA (6/2017 ABSSSI, } \\
10 / 2019 \text { CAP) }\end{array}$ & \multirow[t]{2}{*}{ Fluoroquinolone } & \multirow[t]{2}{*}{$\begin{array}{l}\text { Intravenous and oral } \\
\text { (Melinta, Menarini) }\end{array}$} & \multirow[t]{2}{*}{ ABSSSI, CAP } & \multirow[t]{2}{*}{ O } & \multirow[t]{2}{*}{0} & \multirow[t]{2}{*}{0} & \multirow[t]{2}{*}{$\bullet$} & \multirow[t]{2}{*}{-} & \multirow[t]{2}{*}{-} & \multirow[t]{2}{*}{-} & \multirow[t]{2}{*}{-} \\
\hline & MAA & & & & & & & & & & & \\
\hline $\begin{array}{l}\text { Vaborbactam } \\
\text { and meropenem } \\
\text { (Vabomere) }\end{array}$ & EMA (11/2018) & $\begin{array}{l}\text { Boronate BLI } \\
\text { and carbapenem }\end{array}$ & $\begin{array}{l}\text { Intravenous } \\
\text { (Melinta, Menarini) }\end{array}$ & cUTI & 0 & O & $\mathbf{O}^{\mathrm{b}}$ & I & $\sim$ & $\checkmark$ & - & - \\
\hline Plazomicin (Zemdri) & $\operatorname{FDA}(7 / 2018)$ & Aminoglycoside & Intravenous (Pliva) & cUTI & 0 & O & $\bullet$ & l & - & - & - & - \\
\hline \multirow{2}{*}{$\begin{array}{l}\text { Eravacycline } \\
\text { (Xerava) }\end{array}$} & FDA $(8 / 2018)$ & \multirow[t]{2}{*}{ Tetracycline } & \multirow{2}{*}{$\begin{array}{l}\text { Intravenous } \\
\text { (Tetraphase) }\end{array}$} & \multirow[t]{2}{*}{ clAI } & $?$ & 0 & ○ & l & - & - & - & - \\
\hline & $\operatorname{EMA}(9 / 2018)$ & & & & & & & & & & & \\
\hline $\begin{array}{l}\text { and imipenem } \\
\text { and cilastatin } \\
\text { (Recarbrio) }\end{array}$ & MAA & $\begin{array}{l}\text { carbapenem } \\
\text { and degradation } \\
\text { inhibitor }\end{array}$ & & & & & & & & & & \\
\hline Lefamulin (Xenleta) & FDA (8/2019) & Pleuromutilin & Intravenous and oral & CAP & I & I & I & - & $\sim$ & $\boldsymbol{J}^{c}$ & - & - \\
\hline & MAA & & & & & & & & & & & \\
\hline Cefiderocol & FDA (11/2019) & Siderophore & Intravenous (Shionogi) & cUTI & $\bullet$ & $\bullet$ & $\bullet$ & I & $\sim$ & - & - & - \\
\hline (Fetroja) & MAA & & & & & & & & & & & \\
\hline
\end{tabular}

ABSSSI, acute bacterial skin and skin structure infections; BLI, $\beta$-lactamase inhibitor; CAP, community-acquired pneumonia; CC, new chemical class; $c$ AAl, complicated intra-abdominal infections; CRAB, carbapenem-resistant Acinetobacter baumannii; CRE, third-generation cephalosporin-resistant and carbapenem-resistant Enterobacterales; CRPA, carbapenem-resistant Pseudomonas aeruginosa; cUTI, complicated urinary tract infection; DBO, diazabicyclooctane; EMA, European Medicines Agency; MAA, Marketing Authorization Application (EMA); MoA, new mode of action; NCR, no cross-resistance to other antibiotic classes; PBP, penicillin-binding protein; T, new target. ${ }^{\mathrm{a} O}$ Other pathogens, mostly Gram-positive and/or Neisseria gonorrhoeae. ${ }^{\mathrm{b}}$ Active against Klebsiella pneumoniae carbapenemase (KPC)-producing but not metallo- $\beta$-lactamase-producing Enterobacterales. 'First systemic formulation of this class in humans, still used in animals and topically in humans. - active; ?, possibly active; $\bigcirc$, not or insufficiently active; /, activity not assessed; $\checkmark$, criterion fulfilled; $\sim$, inconclusive data or no agreement among experts; -, criterion not fulfilled. 
and KPC-3, producers. Whether those theoretical advantages compared with piperacillin-tazobactam will translate into improved clinical efficacy in the therapy of ESBL-producing Enterobacterales (not CRE) remains to be proven in randomized controlled clinical trials.
In general, the new BLI combinations contribute to improved options to treat KPC-producing and OXA-48producing Enterobacterales, especially Klebsiella spp., in countries where these are the prevailing resistance mechanisms (FIG. 2). In countries where other $\beta$-lactamases

Table 2 | Antibacterial agents in clinical development (modified according to the WHO clinical pipeline report ${ }^{3}$ )

\begin{tabular}{|c|c|c|c|c|c|c|c|c|c|c|c|}
\hline \multirow[t]{2}{*}{ Name (synonym) } & \multirow[t]{2}{*}{ Phase } & \multirow[t]{2}{*}{ Antibiotic class } & \multirow[t]{2}{*}{$\begin{array}{l}\text { Route of administration } \\
\text { (developer) }\end{array}$} & \multicolumn{4}{|c|}{$\begin{array}{l}\text { Expected activity } \\
\text { against }\end{array}$} & \multicolumn{4}{|c|}{ Characteristics } \\
\hline & & & & 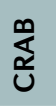 & $\frac{\overleftarrow{\Omega}}{\text { 花 }}$ & 岕 & 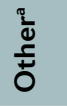 & 过 & ن & $\vdash$ & 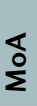 \\
\hline $\begin{array}{l}\text { Sulopenem (intravenous), } \\
\text { Sulopenem etzadroxil and } \\
\text { Probenecid (oral) }\end{array}$ & 3 & Penem & Intravenous and oral (Iterum) & O & O & $\mathrm{O}^{\mathrm{b}}$ & I & - & - & - & - \\
\hline $\begin{array}{l}\text { Durlobactam (ETX2514) } \\
\text { and sulbactam }\end{array}$ & 3 & $\begin{array}{l}\text { DBO-BLI (PBP2 inhibitor) and } \\
\beta \text {-lactam-BLI (PBP1 and PBP3 inhibitor) }\end{array}$ & Intravenous (Entasis) & $\bullet$ & I & I & l & - & - & - & - \\
\hline $\begin{array}{l}\text { Taniborbactam } \\
\text { (VNRX-5133) and Cefepime }\end{array}$ & 3 & Boronate-BLI and cephalosporin & Intravenous (VenatoRx) & O & $?$ & $\bullet$ & l & $\sim$ & $\boldsymbol{J}^{c}$ & - & - \\
\hline $\begin{array}{l}\text { Enmetazobactam (AAl101) } \\
\text { and Cefepime }\end{array}$ & 3 & $\beta$-lactam-BLI and cephalosporin & Intravenous (Allecra) & O & O & $O^{d}$ & l & - & - & - & - \\
\hline Zoliflodacin & 3 & $\begin{array}{l}\text { Topoisomerase inhibitor } \\
\text { (Spiropyrimidenetrione) }\end{array}$ & Oral (Entasis/GARDP) & l & I & I & $\bullet$ & $\checkmark$ & $\checkmark$ & - & $\checkmark$ \\
\hline Gepotidacin & 3 & $\begin{array}{l}\text { Topoisomerase inhibitor } \\
\text { (Triazaacenaphthylene) }\end{array}$ & Intravenous and oral (GSK) & I & I & I & • & $\sim$ & $\checkmark$ & - & $\checkmark$ \\
\hline Contezolid acefosamil & $2 / 3^{f}$ & Oxazolidinone & Intravenous and oral (MicuRx) & I & l & I & 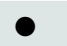 & - & - & - & - \\
\hline Afabicin (Debio-1450) & 2 & Fabl inhibitor & $\begin{array}{l}\text { Intravenous and oral } \\
\text { (Debiopharm) }\end{array}$ & I & I & I & • & $\checkmark$ & $\checkmark$ & $\checkmark$ & $\checkmark$ \\
\hline BOS-228 (LYS228) & 2 & Monobactam & $\begin{array}{l}\text { Intravenous (Boston } \\
\text { Pharmaceuticals) }\end{array}$ & O & O & $\bullet$ & I & - & - & - & - \\
\hline Nafithromycin (WCK-4873) & 2 & Macrolide & Oral (Wockhardt) & l & I & I & ○ & - & - & - & - \\
\hline TNP-2092 & 2 & Rifamycin-quinolizinone conjugate & Intravenous and oral (TenNor) & I & I & I & $?$ & - & - & - & - \\
\hline Zidebactam and Cefepime & 1 & $\begin{array}{l}\text { DBO-BLI (PBP2 inhibitor) and } \\
\text { cephalosporin }\end{array}$ & Intravenous (Wockhardt) & O & $?$ & $\bullet$ & I & - & - & - & - \\
\hline $\begin{array}{l}\text { Nacubactam and } \\
\text { Meropenem }\end{array}$ & 1 & $\begin{array}{l}\text { DBO-BLI (PBP2 inhibitor) and } \\
\text { carbapenem }\end{array}$ & $\begin{array}{l}\text { Intravenous (NacuGen } \\
\text { Therapeutics) }\end{array}$ & O & O & $\boldsymbol{\bullet}^{\mathrm{e}}$ & I & - & - & - & - \\
\hline ETX0282 and Cefpodoxime & 1 & $\begin{array}{l}\text { DBO-BLI (PBP2 inhibitor) and } \\
\text { cephalosporin }\end{array}$ & Oral (Entasis) & O & O & $\bullet^{\mathrm{e}}$ & I & - & - & - & - \\
\hline VNRX-7145 and Ceftibuten & 1 & Boronate-BLI and cephalosporin & Oral (VenatoRx) & O & O & $\bullet^{\mathrm{e}}$ & I & $\sim$ & $\boldsymbol{J}^{c}$ & - & - \\
\hline SPR741 and $\beta$-lactam & 1 & Polymyxin (potentiator) and $\beta$-lactam & Intravenous (Spero) & $?$ & $?$ & $?$ & l & - & - & - & - \\
\hline SPR206 & 1 & Polymyxin & Intravenous (Spero) & $\bullet$ & $\bullet$ & $\bullet$ & l & - & - & - & - \\
\hline SPR720 & 1 & GyrB inhibitor & Oral (Spero) & I & l & I & $\mathbf{O}^{9}$ & $\sim$ & $\checkmark$ & $\sim$ & $\sim$ \\
\hline KBP-7072 & 1 & Tetracycline & Oral (KBP BioSciences) & O & O & O & $\bullet$ & - & - & - & - \\
\hline TP-271 & 1 & Tetracycline & $\begin{array}{l}\text { Intravenous and oral } \\
\text { (Tetraphase) }\end{array}$ & $?$ & O & O & $\bullet$ & - & - & - & - \\
\hline TP-6076 & 1 & Tetracycline & Intravenous (Tetraphase) & $\bullet$ & 0 & $?$ & I & - & - & - & - \\
\hline EBL-1003 (Apramycin) & $1^{\mathrm{h}}$ & Aminoglycoside & Intravenous (Juvabis) & $?$ & - & $?$ & I & - & - & - & - \\
\hline TNP-2198 & 1 & Rifamycin-nitroimidazole conjugate & Oral (TenNor) & I & I & l & $\bullet$ & - & - & - & - \\
\hline TXA709 & 1 & FtsZ inhibitor & Oral and intravenous (Taxis) & 0 & 0 & 0 & ○ & $\checkmark$ & $\checkmark$ & $\checkmark$ & $\checkmark$ \\
\hline $\begin{array}{l}\text { ARX-1796 (oral Avibactam } \\
\text { prodrug) }\end{array}$ & 1 & DBO-BLI and $\beta$-lactam & Oral (Arixa Pharmaceuticals) & O & O & $\bullet^{\mathrm{e}}$ & I & - & - & - & - \\
\hline \multicolumn{12}{|c|}{ 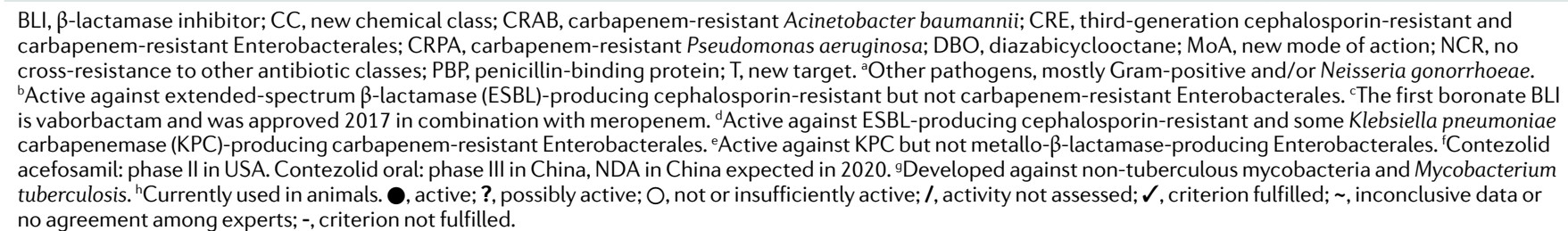 } \\
\hline
\end{tabular}


Table 3 | Activity of $\beta$-lactamase inhibitors in combination (modified according to the WHO clinical pipeline report ${ }^{3}$ )

\begin{tabular}{|c|c|c|c|c|c|c|c|c|c|}
\hline \multirow{2}{*}{$\begin{array}{l}\beta \text {-lactamase inhibitor (synonym, } \\
\text { chemical class) }\end{array}$} & \multirow{2}{*}{$\begin{array}{l}\text { Combination } \\
\text { partner }\end{array}$} & \multirow{2}{*}{$\begin{array}{l}\text { Development } \\
\text { phase }\end{array}$} & \multirow{2}{*}{$\begin{array}{l}\text { ESBL-E } \\
\text { Class A } \\
\text { (ESBL) }\end{array}$} & \multicolumn{4}{|l|}{ CRE } & \multirow[t]{2}{*}{ CRAB } & \multirow[t]{2}{*}{ CRPA } \\
\hline & & & & $\begin{array}{l}\text { Class A } \\
\text { (KPC) }\end{array}$ & $\begin{array}{l}\text { Class D } \\
\text { (OXA-48) }\end{array}$ & $\begin{array}{l}\text { Class B } \\
\text { (NDM) }\end{array}$ & PBP2 & & \\
\hline Durlobactam (ETX2514; DBO) & Sulbactam & 3 & I & l & I & I & $\bullet$ & ? & l \\
\hline $\begin{array}{l}\text { Enmetazobactam (AAl101; penicillanic } \\
\text { acid sulfone) }\end{array}$ & Cefepime & 3 & $\bullet$ & $?$ & 0 & 0 & - & 0 & O \\
\hline Taniborbactam (VNRX-5133; boronate) & Cefepime & 3 & $\bullet$ & - & $\bullet$ & $\bullet$ & - & 0 & $?$ \\
\hline Avibactam (DBO) & Aztreonam & 3 & $\bullet$ & $\bullet$ & $\bullet$ & $\bullet$ & O & O & O \\
\hline Zidebactam (DBO) & Cefepime & 1 & $\bullet$ & - & $\bullet$ & $?$ & $\bullet$ & 0 & $?$ \\
\hline Nacubactam (DBO) & Meropenem & 1 & $\bullet$ & $\bullet$ & $\bullet$ & $?$ & $\bullet$ & 0 & 0 \\
\hline ETX0282 (DBO) & $\begin{array}{l}\text { Cefpodoxime } \\
\text { (oral application) }\end{array}$ & 1 & $\bullet$ & $\bullet$ & $\bullet$ & O & $\bullet$ & 0 & 0 \\
\hline VNRX-7145 (boronate) & $\begin{array}{l}\text { Ceftibuten (oral } \\
\text { application) }\end{array}$ & 1 & $\bullet$ & $\bullet$ & $\bullet$ & 0 & - & 0 & O \\
\hline ARX-1796 (DBO; oral avibactam prodrug) & $\begin{array}{l}\text { To be determined } \\
\text { (oral application) }\end{array}$ & 1 & $?$ & $?$ & $?$ & O & 0 & 0 & 0 \\
\hline
\end{tabular}

Activity of $\beta$-lactamase inhibitors in combination against representative $\beta$-lactamases in Enterobacterales, binding to PBP2 in Enterobacterales, and antibacterial activity of the BLI combination against critical priority pathogens. CRAB, carbapenem-resistant Acinetobacter baumannii; CRPA, carbapenem-resistant Pseudomonas aeruginosa; CRE, carbapenem-resistant Enterobacterales; DBO, diazabicyclooctanes; ESBL-E, extended-spectrum $\beta$-lactamase-producing third-generation cephalosporin-resistant Enterobacterales; PBP2, penicillin-binding protein 2. 0 , active; ?, possibly active; $O$, not or insufficiently active; /, activity not assessed; - , no inhibitory activity.

Siderophore

Small, high-affinity iron-chelating compounds that are secreted by microorganisms and serve primarily to transport iron across cell membranes. dominate, they hardly make a difference to existing antibacterial drugs. Owing to at least some pre-existing cross-resistance, all these new $\beta$-lactam antibiotics and BLI combinations should only be used with confirmed susceptibility results. This drug class has been and will continue to be used heavily, and hence, continued selection pressure through the new derivatives of this group will quickly lead to expected but also unexpected resistances. The first signs of new resistance developments in Klebsiella spp. are already visible: ceftazidime-avibactam is vulnerable to KPC $\beta$-lactamase mutants with increased hydrolytic capacity for ceftazidime. Such mutants can easily be obtained in vitro ${ }^{29}$ and have been selected in patients treated with ceftazidime-avibactam ${ }^{58}$. For ceftolozane-tazobactam, there are reports of in vivo selection of $P$. aeruginosa mutants with sequence mutations in AmpC that confer resistance to both ceftolozanetazobactam and ceftazidime-avibactam ${ }^{31,32}$. Many $\beta$-lactamase-encoding genes travel together on mobile elements with transmissible resistance factors for other antibacterial classes ${ }^{59}$. Selection pressures due to various antibiotic classes and the spread of such resistant bacteria may leave the new BLI combinations a short period of useful activity. Though each of the BLIs has its own inhibitory pattern, they may not translate into phenotypic differences in the MIC values of the combinations. However, good regional surveillance of molecular information regarding the most common $\beta$-lactamases and non-enzymatic mechanisms (porin deficiency and efflux), as well as broad antibiotic knowledge, will be essential to making informed decisions and the best use of the new BLI combinations in specific health-care environments ${ }^{60}$.

New stand-alone $\beta$-lactam antibiotics. Cefiderocol is a cephalosporin structurally related to ceftazidime and cefepime but more stable to various $\beta$-lactamases. The cephalosporin molecule is linked to a siderophore that can bind to iron, which facilitates bacterial cell entry in addition to the usual entry via porin channels; this results in improved penetration of cefiderocol in the bacterial cell, which is most relevant in non-fermenters ${ }^{61,62}$. In Enterobacterales, variations in iron transport channel expression, which varies by species and within species, may cause a wide distribution of MICs ${ }^{63}$. Compared with ceftazidime-avibactam, cefiderocol has similar in vitro microbiological activity against Enterobacterales and P. aeruginosa, but it is more active against Acinetobacter, Stenotrophomonas and Burkholderia species. The resistance prevalence in CRE and CRPA is a little lower than with ceftazidime-avibactam ${ }^{64}$. The main weakness of cefiderocol is its limited activity against NDM-producing E. coli as well as OXA-23-producing and OXA-24producing Acinetobacter $\mathrm{spp}^{65}$. $\mathrm{MIC}_{90}$ values are about eight doubling dilutions higher in KPC-2-producing Enterobacterales than in wild-type strains. Due to the very low MICs in wild-type strains, the reduction of activity may still keep cefiderocol MICs for many strains below the clinical breakpoint ${ }^{63}$. Similarly, the reduced susceptibility of ESBL-producing, carbapenemaseproducing or AmpC-producing strains may not translate into immediate clinical resistance ${ }^{63}$ but may be problematic in critically ill patients with altered pharmacokinetic profiles. However, the most problematic XDR or PDR strains have variable susceptibility, with $\sim 64 \%$ of NDMproducing pathogens testing as susceptible ${ }^{65}$. MICs clustering around the breakpoint may be the beginning of a stepwise development of resistance, and reliable susceptibility testing will be essential.

Cefiderocol has recently been approved by the FDA for the treatment of complicated UTIs (including pyelonephritis) caused by susceptible Gram-negative bacteria in patients with limited or no alternative treatment options ${ }^{66}$. However, the results from a small descriptive study in critically ill patients with XDR Gram-negative bacteria have raised concerns, as higher rates of 
infection-related and non-infection-related deaths with treatment failure were seen for cefiderocol than for the comparator (best available therapy). In addition, the development of cefiderocol resistance during therapy was linked to poor outcomes. The results of this study, with its inherent limitations of a small descriptive trial, leave clinicians with little evidence to support the use of cefiderocol in patients with XDR Gram-negative bacteria, especially Acinetobacter spp. and Pseudomonas spp. ${ }^{67}$. A better understanding of cefiderocol efficacy and the associated risk factors will require additional well-designed studies.

BOS-228 is a new monobactam in phase II clinical development. Like other monobactams, BOS-228 inhibits PBP3 and is stable to MBLs. Additionally, it has been modified to resist hydrolysis by most serine $\beta$-lactamases in Enterobacteriaceae ${ }^{68}$. Cross-resistance exists with aztreonam when non- $\beta$-lactamase mechanisms are involved (for example, upregulated efflux and/or porin downregulation, or mutations in PBP3 and the envelope stress response system) ${ }^{69}$.

Sulopenem and tebipenem, in the form of bioavailable esters, are both in phase III clinical development and will provide new oral therapies for ESBL-producing (MDR) Enterobacteriaceae, but they also confront the medical community with specific challenges. Sulopenem is a thiopenem with intravenous and oral formulations

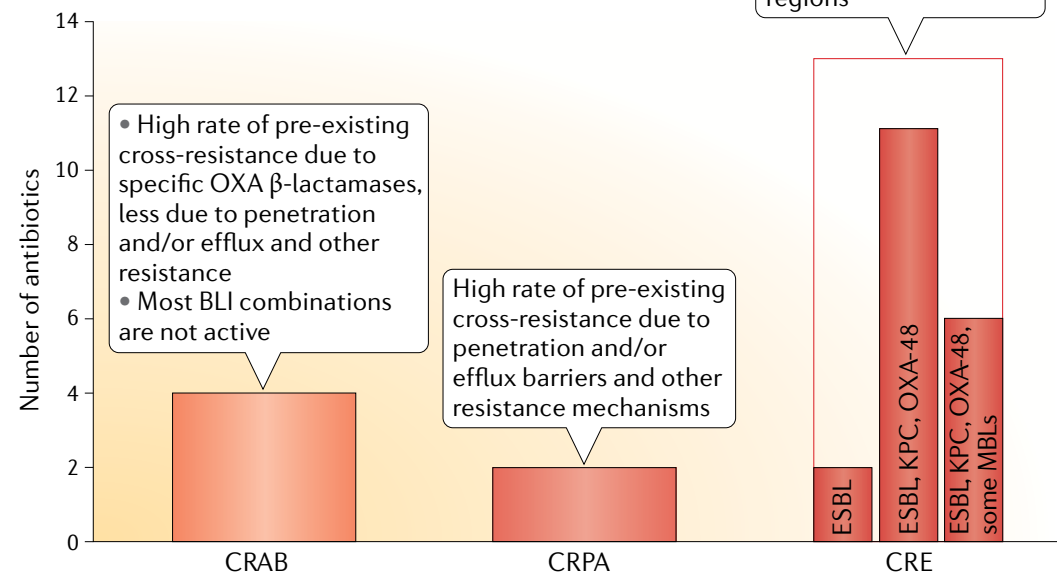

Fig. 2 | Numbers of antibiotics in clinical development or approved since 2017 with activity against the critical priority pathogens CRAB, CRPA and CRE. The most recently approved or currently developed new antibiotics have focused on carbapenemresistant Enterobacterales (CRE), but cover them incompletely and dependent on the geographic distribution of resistance determinants. These CRE antibiotics may cover bacteria that produce only extended-spectrum $\beta$-lactamases (ESBL), or may have added activity against KPC and OXA-48 enzymes, or even additional activity against some metallo- $\beta$-lactamases (MBL). Carbapenem-resistant Acinetobacter baumannii (CRAB) has multiple intrinsic and acquired resistance mechanisms, which causes high rates of extensive drug resistance and pan drug resistance. The efficacy of the few new antibiotics with activity against Acinetobacter spp. is limited, owing to pre-existing reduced susceptibility or non-susceptibility in the most resistant strains. The rate of pre-existing cross-resistance in carbapenem-resistant $P$ seudomonas aeruginosa (CRPA) is high, and no substantial solution will be provided by new antibiotics. BLI, $\beta$-lactamase inhibitor. (prodrug), whereas tebipenem is an orally available carbapenem (as a prodrug) that was approved in Japan in 2009 for the treatment of paediatric respiratory tract infections (therefore, it is not included in TABLE 2). Both antibiotics have similar spectra against Gram-negative bacteria compared with ertapenem, which focuses on ESBL-producing Enterobacteriaceae. These agents show complete cross-resistance with ertapenem and other carbapenems ${ }^{70}$. Sulopenem failed to demonstrate non-inferiority compared with ertapenem for complicated intra-abdominal infections in a recent phase III trial $^{71}$. Sulopenem and tebipenem were developed for community-acquired uncomplicated UTIs and for oral follow-on after intravenous therapy for complicated $\mathrm{UTIs}^{72,73}$. If used widely, they will most likely exert a strong selection pressure for carbapenem resistance, like any other carbapenem ${ }^{74}$. Antibiotic stewardship programmes would need to be established in the community to mitigate this risk. From the public health perspective, oral carbapenem-sparing options for the treatment of MDR strains would be preferable.

\section{Tetracycline derivatives}

New semisynthetic and synthetic tetracycline derivatives, based on new chemistry approaches, have been recently approved or are in clinical development. Tetracyclines were discovered in the 1940s and have been widely used in human and veterinary medicine and in agricultural applications, which has contributed to the widespread dissemination of tetracycline resistance. Efflux pumps and ribosome protection proteins are the most common resistance mechanisms ${ }^{75}$. In Gram-negative bacteria, target-site mutations have been described, recently even for tigecycline ${ }^{76}$. Lately, enzymatic degradation has gained more attention, as new, highly transferrable plasmid-mediated tetracycline-inactivating enzymes, Tet(X3) and Tet(X4), have been described and are globally present. They degrade all tetracyclines, including tigecycline and the recently approved eravacycline and omadacycline ${ }^{77-79}$. Such plasmids may carry other resistance determinants, such as $m c r l$ (colistin resistance) and genes encoding MBLs (for example, NDM), thus potentially rendering isolates $\mathrm{XDR}^{78,80}$. The fully synthetic eravacycline (intravenous) has activity against CRE comparable to tigecycline and exhibits cross-resistance with tigecycline ${ }^{79,81}$. The semisynthetic minocycline derivative omadacycline has been recently approved for the oral and intravenous treatment of acute bacterial skin and skin structure infections (ABSSSI) and community-acquired pneumonia (CAP). The activity against XDR Enterobacterales is insufficient ${ }^{82}$.

Three more tetracyclines are in early clinical development: first is KBP-7022, with a spectrum similar to tigecycline $^{83,84}$ but lower MICs in Acinetobacter spp. ${ }^{85}$. No information is available about cross-resistance with tigecycline. The second tetracycline is TP-6076, with activity against Enterobacterales and Acinetobacter spp. and lower MICs than tigecycline. The impact of these in vitro findings is not known yet, pending pharmacokinetic-pharmacodynamic studies and breakpoint decisions. In tigecycline-non-susceptible and minocycline-non-susceptible Acinetobacter isolates, 
the MICs of TP-6076 were slightly increased ${ }^{86}$. In K. pneumoniae, tigecycline-resistant strains are mostly also resistant to $\mathrm{TP}-6076\left(\mathrm{REF}^{87}\right)$, and resistance to tigecycline in carbapenem-resistant strains is high in some areas ${ }^{27}$. Resistance in K. pneumoniae is due to overexpressed RamA, a transcriptional regulator that modulates efflux pump expression ${ }^{88}$. Such a resistance mechanism is increasingly found in CRE with porin deficiency, production of various $\beta$-lactamases and aminoglycoside-inactivating enzymes ${ }^{28}$, thus reducing the value of this antibiotic as a treatment option for the most resistant bacteria. It is not yet known whether the liabilities of tigecycline, including low plasma exposure, concentration-independent plasma protein binding and adverse-event concerns, will be mirrored by the new tetracyclines ${ }^{89}$. Preliminary information points to an adverse-event profile of TP-6067 similar to that of tigecycline, with dose-dependent gastrointestinal side effects, including nausea and vomiting as the most frequently reported adverse effects. The third tetracycline - TP-271, a synthetic tetracycline - is very similar to tigecycline and is active against respiratory pathogens. It is not affected by Tet(M) (ribosomal protection protein), but is affected by Tet(A) (efflux) and Tet(X) (enzymatic inactivation $)^{90}$.

Similar to other derivatives of long-used classes, the new tetracyclines address some class-specific resistance mechanisms of the older representatives of this class, such as doxycycline, but have a high degree of cross-resistance to tigecycline. They do not represent reliable alternatives for XDR or PDR Gram-negative bacteria. Potential benefits over tigecycline regarding non-potency-related characteristics, such as pharmacokinetics and safety, have not been shown so far.

\section{Other derivatives of old classes}

In addition to $\beta$-lactams, $\beta$-lactamase inhibitors and tetracyclines, a few other old classes have been used as starting points to develop derivatives. The polymyxins are seeing a revival with SPR206, a polymyxin derivative with slightly improved potency that also reduces the magnitude of cross-resistance. The increasingly described plasmid-mediated mcr resistance is often associated with other resistance genes that encode for $\beta$-lactamases (including carbapenemase) and for resistances to other antibacterial classes, which may include fluoroquinolones and aminoglycosides ${ }^{91}$. Thus, several antibiotic classes may select for XDR or even PDR strains. Carbapenem-resistant K. pneumoniae, which also exhibited resistance to colistin in more than $30 \%$ of the strains, has been described in a recent study ${ }^{28}$. It is not clear yet how polymyxin resistance and associated cross-resistance to unrelated classes will develop over the next years, whether SPR206 will be of any benefit in polymyxin-resistant strains and whether the promise of lower class-specific toxicity will hold true in patients ${ }^{92}$.

Another polymyxin derivative (SPR741), with limited intrinsic activity but improved safety, is being studied for permeabilization of the outer membrane, thereby granting antibacterial agents access to their intracellular targets ${ }^{93}$. The most beneficial combinations of such a 'potentiator' strategy are not known yet, and development is pending.
The aminoglycoside class has seen a recent addition, plazomicin, to reduce the impact of aminoglycosidemodifying enzymes. Although plazomicin has activity against many CRE isolates, organisms that produce the NDM-1 MBL or OXA-48 enzymes are often resistant to plazomicin, due to co-production of ribosomal methyl transferase $e^{94,95}$. Another aminoglycoside has also entered its first trials in humans. This aminoglycoside, apramycin, has been licensed since 1980 for oral use in veterinary medicine. It was active in $87 \%$ of carbapenem-resistant Klebsiella isolates from Greek hospitals, a susceptibility rate similar to that for plazomicin. Though apramycin is not affected by $16 \mathrm{~S}$ rRNA methylases that cause resistance to plazomicin and all other aminoglycosides, it is ineffective against the acetyltransferase AAC(3)-IV-producing strains ${ }^{96,97}$. Resistance in human isolates was already described in $1993\left(\mathrm{REF}^{98}\right)$. Its usefulness against XDR Gram-negative strains will depend on the future epidemiology of aminoglycoside resistance, but rapid resistance development and spread could be anticipated if apramycin were to be used clinically.

Derivatives of old classes with activity against Grampositive bacteria are in clinical development. No information on their differentiation from the drugs already available has been published concerning the oxazolidinone contezolid, with equal activity and non-inferiority compared with linezolid ${ }^{99}$, and the ketolide nafithromycin, which is comparable to telithromycin ${ }^{100}$. Two rifamycin conjugates, TNP-2092 (rifamycin-quinolizinone conjugate with rifampicin-like activity $\left.{ }^{101}\right)$ and TNP-2198 (rifamycin-nitroimidazole conjugate) are in clinical development. The published information has been insufficient to assess the potential clinical usefulness of these rifamycin conjugates.

\section{Beyond old classes}

New chemical scaffolds, new targets or binding sites, and a new mode of action. Two new antibiotics that represent new chemical scaffolds with activity against Gram-positive bacteria are in clinical development. The target FabI, an enoyl-ACP reductase that is the ratelimiting enzyme in the last step for fatty-acid biosynthesis, is known from existing FabI inhibitors (isoniazid, Mycobacterium tuberculosis) and triclosan (in some consumer products) ${ }^{102}$. Afabicin (Debio-1450) is a new intravenously and orally administered FabI inhibitor that exhibits selective antibacterial activity against staphylococcal species ${ }^{103}$. As expected, there is no cross-resistance with other antibacterial drugs used for staphylococcal infections ${ }^{104}$. Preliminary studies have indicated that afabicin may not be prone to rapid emergence of resistance, despite binding to a single target, possibly due to its high-affinity binding ${ }^{102}$. It is currently being developed for the treatment of bone and joint infections ${ }^{105}$. Other FabI inhibitors are in preclinical development (for example, MUT056399) ${ }^{106}$.

The prodrug TXA709 is a benzamide compound and targets the bacterial protein FtsZ, which has an essential role in septum formation and prevents bacterial cell division without a eukaryotic homologue ${ }^{107}$. Its activity is focused on $S$. aureus, with no pre-existing cross-resistance to commonly used antibiotics, due to its 
DNA gyrase

An enzyme within the class

of topoisomerases that has multiple roles in DNA

replication, recombination

and transcription.

Topoisomerase

An enzyme that participates

in the overwinding or

underwinding of DNA. new chemical class, new target and new mode of action. As expected, this single-target agent leads to a relatively high frequency of resistance, which may be mitigated by using it in combination with other agents ${ }^{108}$. Though the immediate medical need for a new drug against multiresistant $S$. aureus is low, a new-class antibacterial drug may be valuable in selected cases and on a broader basis in the future.

The topoisomerase inhibitors zoliflodacin (spiropyrimidinetrione) and gepotidacin (triazaacenaphthylene) are new chemical scaffolds and bind to gyrase, the same target as for the fluoroquinolones, but at distinct binding sites. They were developed for the treatment of uncomplicated urogenital gonorrhoea. Due to its different chemical structure and distinct binding site, zoliflodacin has so far not shown to be cross-resistant to fluoroquinolones ${ }^{109}$. For gepotidacin, a phase II study in uncomplicated urogenital gonorrhoea raised some questions regarding cross-resistance to ciprofloxacin and the emergence of resistance. Three isolates with higher gepotidacin MICs were quinolone resistant and showed a mutation in the parC gene, which is known to affect gepotidacin binding. Two of these isolates developed high-level resistance to gepotidacin and were bacteriological failures in the clinical trial ${ }^{110}$. Due to overlapping binding sites, gepotidacin may show some cross-resistance to ciprofloxacin, and resistance may soon emerge once it is used in clinical practice. Gepotidacin is currently also being developed for uncomplicated UTIs. Its MICs against fluoroquinolone-susceptible E. coli are much higher than for levofloxacin, but cross-resistance to fluoroquinolones has not been described yet in $E$. coli $i^{111,112}$.

Although fluoroquinolones target both the DNA gyrase GyrA subunit and the topoisomerase IV ParC subunit, no inhibitor of GyrB and/or ParE is currently in clinical use ${ }^{113}$. The new GyrB inhibitor SPR-720 (aminobenzimidazole) inhibits the ATPase activity of gyrase in $M$. tuberculosis and non-tuberculous mycobacteria $(\mathrm{NTM})^{114}$. It will be developed for these bacteria.

\section{Box 1 Antibacterial drugs in clinical development and future perspectives}

- The clinical pipeline is dominated by derivatives of most major known chemical and functional classes, especially $\beta$-lactams and $\beta$-lactamase inhibitors (BLIs), but also tetracyclines.

- All antibiotic candidates currently under development to treat infections caused by the WHO critical-priority bacteria have at least some cross-resistance with existing agents.

- Selected class-specific resistance mechanisms are being addressed, others remain unaffected.

- The rate of cross-resistance is relatively high in extensively drug-resistant (XDR) strains and specifically in pan-drug-resistant (PDR) strains.

- The pipeline focused on carbapenem-resistant Enterobacterales (CRE) has incomplete $\beta$-lactamase coverage, especially for $\beta$-lactamases prevalent in Asia and Africa, but also in some European countries.

- Susceptibility rates depend on the epidemiology of resistance mechanisms in different regions and locations.

- The best use of new drugs is achieved if the regional molecular resistance epidemiology is known and if agents are selected according to susceptibility tests.

- Few innovative new antibiotics are being developed against Staphylococcus aureus, as well as Neisseria gonorrhoeae and Clostridioides difficile.

- There is a continued high need for innovation, especially for new-class antibiotics without pre-existing cross-resistance.
The development of intravenous murepavidin, a cyclic peptide that targets the lipopolysaccharide transport protein $\mathrm{D}(\mathrm{LptD})$ in $P$. aeruginosa, was terminated in July 2019 owing to concerns about nephrotoxicity observed in phase III. The inhaled form of murepavidin is in preclinical development for $P$. aeruginosa infections in patients with cystic fibrosis. Therefore, it is not included in TABLE 2.

Lefamulin, a member of the pleuromutilin class, was approved by the FDA for the treatment of communityacquired bacterial pneumonia in 2019 (REF. ${ }^{115}$ ). Though pleuromutilins are an established class for systemic use in veterinary medicine and have been used topically in humans (retapamulin was approved in 2007), lefamulin represents a new scaffold for systemic use in humans. Whether the prior use and selection pressure of this class will accelerate the emergence of resistance against lefamulin remains to be seen. Caution should be exercised when prescribing this drug to patients with QT prolongation and ventricular arrhythmias. It has shown embryo-fetal toxicity in animals and should not be prescribed to pregnant women and females of reproductive potential without effective contraception ${ }^{115}$.

\section{New antibacterial agents against Clostridioides difficile.}

New antibacterial drugs against $C$. difficile infection are being developed with the goal of reducing recurrences. These agents are usually orally available and not absorbed, and thus systemic pharmacokinetics and toxicity do not represent major challenges in the discovery process. This group of antibiotics has been included because they represent mainly new chemical classes with new targets and new modes of action. The most advanced compound is ridinilazole, a bis-benzimidazole that has been suggested to inhibit cell division and is associated with reduction in spore and toxin production ${ }^{116,117}$. Three new chemical structures with new targets and new modes of action are in early clinical development. MGB-BP3 is a distamycin derivative and binds to the DNA minor groove ${ }^{118}$. It acts on multiple binding sites and interferes with transcription. ACX-362E is a synthetic purine and targets DNA polymerase IIIC ${ }^{119}$. CRS-3123 is a diaryldiamine derivative that inhibits the Met-aminoacyl-tRNA synthetase $^{120}$. Little information is available about the propensity for rapid emergence of single-step resistance due to mutations in the target of CRS-3123. The clinical value of these new drugs will depend on proof that they can reliably reduce the rate of recurrence.

\section{Conclusion}

This narrative overview has critically reviewed the antibacterial agents in clinical development and confirms the limited scope of these new antibacterial agents, especially against Gram-negative critical-priority pathogens. In particular, all agents in development against the critical-priority pathogens exhibit some degree of pre-existing cross-resistance. A summary of our conclusions may be found in BOX 1 .

The global clinical pipeline is dominated by derivatives of known chemical and functional classes. Though the experience with widely used antibacterial classes and familiarity with class-specific safety profiles and 
pharmacokinetic and pharmacodynamic properties are good starting points for antibacterial research and development programmes, chemical modifications lead to improvements that are usually incremental and address only selected class-specific resistance mechanisms that are known at the time of lead optimization, whereas other mechanisms remain unaffected. Thus, a relatively high rate of pre-existing cross-resistance in XDR strains or substantially increased MICs compared with the wild-type strains may limit the benefit of such new therapies in many geographic regions and locations. They are unlikely to ease the global threat of XDR and PDR Gram-negative bacteria. Knowledge of the surveillance data on the distribution of molecular epidemiology and resistance mechanisms at the local level will be an absolute prerequisite for adequate therapy decisions. This fact, and the focus on one or a few specific pathogens, limits these antibacterial drugs for use in the initial treatment phase of critically ill patients, before pathogen and susceptibility profiles are known.

Most new drugs in clinical development are not active against CRAB. The three compounds that are active (a BLI combination, a polymyxin derivative and a tetracycline derivative) are all affected by pre-existing cross-resistance or by substantially increased MICs compared with wild-type strains, which may reduce their efficacy in critically ill patients due to pharmacokinetic and pharmacokinetic-pharmacodynamic variability ${ }^{121}$. In Pseudomonas spp., nonspecific resistance mechanisms such as porin deficiency and overexpressed efflux pumps may lead to a high baseline resistance rate to new derivatives and reduce the usefulness not only of new BLI combinations but also of other antibacterial classes.

In addition to pre-existing cross-resistance in all key bacterial pathogens, new resistance will emerge rapidly to newly introduced derivatives if they are used widely and replace older empiric therapies without adequate stewardship. The emergence of new resistance determinants is also driven by all currently used antibiotics of the old class. Some of these examples have already been described for ceftazidime-avibactam, other BLI combinations, new tetracyclines and plazomicin. Unpredictable components of the evolution of resistance to known antibacterial classes ${ }^{122}$ may increase the risk of unacceptably high rates of resistance to a new derivative that emerge during development or soon thereafter. Though discovering and developing new chemical structures for new bacterial targets is extremely challenging, it is the most promising strategy for starting with an effective antibacterial agent without pre-existing cross-resistance. Substantial efforts should be directed to this endeavour.

The antibacterial drugs currently in phase III clinical development that target Gram-negative bacteria are usually evaluated for the treatment of complicated UTIs, complicated intra-abdominal infections (cIAI), and sometimes hospital-acquired pneumonia (HAP) and ventilator-associated pneumonia (VAP) caused by susceptible bacteria. They are compared to standardof-care antibiotics in non-inferiority clinical trial designs. Though complicated UTI and cIAI caused by susceptible bacteria are not a high unmet medical need, as several antibacterial alternatives exist, this development strategy is aligned with a streamlined regulatory pathway that ensures a more robust safety population. When a new antibacterial drug is approved, clinicians often have minimal information about its efficacy in patients infected with XDR or PDR bacteria, where the medical need is highest, even when clinical trials are designed to include such patients. In addition to the streamlined pivotal studies undertaken to achieve approval, only company-sponsored non-clinical information is available to provide some early indications of potential differentiations between similar drugs. After approval, physicians are faced with a lack of independent critical analysis of the data and evidence, a lack of surveillance systems to rapidly detect new patterns of resistance, a lack of updated therapeutic guidelines and a lack of rapid diagnostic capabilities in many institutions. Nevertheless, these new antibiotics may still be useful for individual patients or specific situations, even without the availability of needed evidence at the time of approval based on careful situation-specific evaluation of the new drug.

All the new-class antibacterial agents focus on Grampositive bacteria (especially $S$. aureus or C. difficile) or Gram-negative cocci (for example, Neisseria gonorrhoeae), thus highlighting the scientific barriers to antibiotic discovery in the field of Gram-negative rods. Overcoming barriers to drug penetration and efflux avoidance for Gram-negative bacteria is still a main hurdle that impedes innovation ${ }^{123}$.

In conclusion, the need for research and development of new antibacterial drugs, especially against the WHO critical-priority pathogens, is still strong. Efforts should focus on innovation and antibacterial agents without pre-existing cross-resistance (that is, on new classes or new targets) in order to provide drugs for the most resistant pathogens and prepare for unpredictable resistance challenges in the future. The discovery of such new antibiotics will require sustained commitment over a long time period; substantial levels of resources will be needed to solve the numerous challenges of new-class antibacterial agents, and collective efforts will be needed to expand the science base.

Published online 9 March 2020
1. Tacconelli, E. et al. Discovery, research, and development of new antibiotics: the WHO priority list of antibiotic-resistant bacteria and tuberculosis. Lancet Infect. Dis. 18, 318-327 (2018).

This WHO list prioritizes target pathogens for antibacterial drug research and discovery with a global perspective.

2. Theuretzbacher, $U$. et al. Analysis of the clinical antibacterial and antituberculosis pipeline. Lancet Infect. Dis. 19, e40-e50 (2019).
3. World Health Organization. 2019 antibacterial agents in clinical development: an analysis of the antibacterial clinical development pipeline. https://apps.who.int/iris clinical development pipeline. https://apps. Who.int/iris/
bitstream/handle/10665/330420/9789240000193bitstream/handle/10665/330420/9789240000193
eng.pdf (WHO, 2019). This WHO report presents the updated analysis of the global clinical pipeline including drugs against tuberculosis.

4. Theuretzbacher, U. Antibiotic innovation for future public health needs. Clin. Microbiol. Infect. 23, 713-717 (2017)
5. Theuretzbacher, U. Global antimicrobial resistance in Gram-negative pathogens and clinical need. Curr. Opin. Microbiol. 39, 106-112 (2017)

6. Theuretzbacher, U. \& Piddock, L. J. V. Non-traditional antibacterial therapeutic options and challenges. Cell Host Microbe 26, 61-72 (2019).

7. Rex, J. H., Fernandez Lynch, H., Cohen, I. G., Darrow, J. J. \& Outterson, K. Designing development programs for non-traditional antibacterial agents. Nat. Commun. 10, 3416-3416 (2019). 
8. J Libardo, M. D., Boshoff, H. I. \& Barry, C. E. III. The present state of the tuberculosis drug development pipeline. Curr. Opin. Pharmacol. 42, 81-94 (2018).

9. World Health Organization. Global tuberculosis report 2019. https://www.who.int/tb/publications/global_ report/en/ (WHO, 2019).

10. Xie, R., Zhang, X. D., Zhao, Q., Peng, B. \& Zheng, J. Analysis of global prevalence of antibiotic resistance in Acinetobacter baumannii infections disclosed a faster increase in OECD countries. Emerg. Microbes Infect. 7, 31 (2018)

11. Wright, M. S. et al. New insights into dissemination and variation of the health care-associated pathogen Acinetobacter baumannii from genomic analysis. mBio 5, e00963-13 (2014).

Acinetobacter shows widespread genetic variation among strains, a high variability in antibiotic resistance determinants and dynamic genomic change over short evolutionary time spans.

12. Asif, M., Alvi, I. A. \& Rehman, S. U. Insight into Acinetobacter baumannii: pathogenesis, global resistance mechanisms of resistance treatment options, and alternative modalities. Infect. Drug. Resist. 11, 1249-1260 (2018)

13. Tuan Anh, N. et al. Molecular epidemiology and antimicrobial resistance phenotypes of Acinetobacter baumannii isolated from patients in three hospitals in southern Vietnam. J. Med. Microbiol. 66, 46-53 (2017).

14. Castanheira, M., Deshpande, L. M., Costello, A., Davies, T. A. ¿ Jones, R. N. Epidemiology and carbapenem resistance mechanisms of carbapenem non-susceptible Pseudomonas aeruginosa collected during 2009-11 in 14 European and Mediterranean countries. J. Antimicrob. Chemother. 69, 1804-1814 (2014).

15. Cabot, G. et al. Overexpression of AmpC and efflux pumps in Pseudomonas aeruginosa isolates from bloodstream infections: prevalence and impact on resistance in a Spanish multicenter study. Antimicrob. Agents Chemother. 55, 1906-1911 (2011).

16. del Barrio-Tofiño, E. et al. Genomics and susceptibility profiles of extensively drug-resistant Pseudomonas aeruginosa isolates from Spain. Antimicrob. Agents Chemother. 61, e01589-17 (2017)

This resistome analysis of extensively drugresistant Pseudomonas reveals the molecular epidemiology and a broad variety of resistance mechanisms.

17. Sader, H. S., Flamm, R. K., Streit, J. M., Doyle, T. B. \& Castanheira, M. Antimicrobial activity of cefepimezidebactam (WCK 5222) against clinical isolates of carbapenem-resistant Enterobacterales collected worldwide in 2018. ASM/ESCMID Drug Development Conference 2019 https://www.jmilabs.com/data/ posters/ASM-ESCMID2019-cefepime-zidebactamCRE pdf (2019)

18. Castanheira, M., Doyle, T. B., Kantro, V., Mendes, R. E. $\S$ Shortridge, D. Meropenem-vaborbactam activity against carbapenem-resistant Enterobacterales isolates collected in U.S. hospitals during 2016-2018. Antimicrob. Agents Chemother. 64, 01951-19 (2019).

19. Dadashi, M. et al. Frequency distribution, genotypes and the most prevalent sequence types of New Delh metallo-beta-lactamase-producing Escherichia coli among clinical isolates around the world; a review. J. Glob. Antimicrob. Resist. 19, 284-293 (2019).

20. Bush, K. \& Bradford, P. A. Epidemiology of betalactamase-producing pathogens. Clin. Microbiol. Rev. 33, e00047-19 (2020)

21. Grundmann, H. et al. Occurrence of carbapenemaseproducing Klebsiella pneumoniae and Escherichia coli in the European survey of carbapenemase-producing Enterobacteriaceae (EuSCAPE): a prospective, multinational study. Lancet Infect. Dis. 17, 153-163 (2017).

22. Mueller, L. et al. Phenotypic, biochemical and genetic analysis of KPC-41, a KPC-3 variant conferring resistance to ceftazidime-avibactam and exhibiting reduced carbapenemase activity. Antimicrob. Agents Chemother. 63, 01111-01119 (2019).

23. Giddins, M. J. et al. Successive emergence of ceftazidime-avibactam resistance through distinct genomic adaptations in bla(KPC-2)-harboring Klebsiella pneumoniae sequence type 307 isolates. Antimicrob. Agents Chemother. 62, e02101-e02117 (2018).

24. Palzkill, T. Structural and mechanistic basis for extended-spectrum drug-resistance mutations in altering the specificity of TEM, CTX-M, and KPC $\beta$-lactamases. Front. Mol. Biosci. 5, 16-16 (2018).
25. Chetri, S. et al. AcrAB-TolC efflux pump system plays a role in carbapenem non-susceptibility in Escherichia coli. BMC Microbiol. 19, 210 (2019).

26. Alm, R. A., Johnstone, M. R. \& Lahiri, S. D. Characterization of Escherichia coli NDM isolates with decreased susceptibility to aztreonam/avibactam: role of a novel insertion in PBP3. $J$. Antimicrob. Chemother. 70, 1420-1428 (2015).

27. Galani, I. et al. Nationwide epidemiology of carbapenem resistant Klebsiella pneumoniae isolates from Greek hospitals, with regards to plazomicin and aminoglycoside resistance. BMC Infect. Dis. 19, 167 (2019).

28. Capone, A. et al. High rate of colistin resistance among patients with carbapenem-resistant Klebsiella pneumoniae infection accounts for an excess of mortality. Clin. Microbiol. Infect. 19, E23-E30 (2013).

29. Andrey, D. O. et al. An emerging clone, Klebsiella pneumoniae carbapenemase 2-producing K. pneumoniae Sequence Type 16, associated with high mortality rates in a CC258-endemic setting. Clin. Infect. Dis. https://doi.org/10.1093/cid/ciz1095 (2019).

30. David, S. et al. Epidemic of carbapenem-resistant Klebsiella pneumoniae in Europe is driven by nosocomial spread. Nat. Microbiol. 4, 1919-1929 (2019).

31. Pérez-Văzquez, M. et al. Emergence of NDM-producing Klebsiella pneumoniae and Escherichia coli in Spain: phylogeny, resistome, virulence and plasmids encoding blaNDM-like genes as determined by WGS J. Antimicrob. Chemother. 74, 3489-3496 (2019).

32. Krajnc, A., Lang, P. A., Panduwawala, T. D., Brem, J. \& Schofield, C. J. Will morphing boron-based inhibitors beat the $\beta$-lactamases? Curr. Opin. Chem. Biol. 50, 101-110 (2019).

33. Tehrani, K. H. M. E. \& Martin, N. I. $\beta$-lactam/ $\beta$-lactamase inhibitor combinations: an update. MedChem Comm 9 , 1439-1456 (2018)

34. Linciano, P., Cendron, L., Gianquinto, E., Spyrakis, F. \& Tondi, D. Ten years with New Delhi metallo-betalactamase-1 (NDM-1): from structural insights to inhibitor design. ACS Infect. Dis. 5, 9-34 (2019).

35. Lomovskaya, O. et al. Vaborbactam: spectrum of beta-lactamase inhibition and impact of resistance mechanisms on activity in Enterobacteriaceae. Antimicrob. Agents Chemother. 61, e01443-17 (2017).

36. Papp-Wallace, K. M. et al. Relebactam is a potent inhibitor of the KPC-2 $\beta$-lactamase and restores imipenem susceptibility in KPC-producing Enterobacteriaceae. Antimicrob. Agents Chemother. 62, e00174-18 (2018)

37. Karlowsky, J. A. et al. In vitro activity of imipenem/ relebactam against Gram-negative ESKAPE pathogens isolated in 17 European countries: 2015 SMART surveillance programme. J. Antimicrob. Chemother 73, 1872-1879 (2018)

38. Balabanian, G., Rose, M., Manning, N., Landman, D. $\&$ Quale, J. Effect of porins and blaKPC expression on activity of imipenem with relebactam in Klebsiella pneumoniae: can antibiotic combinations overcome resistance? Microb. Drug. Resist. 24, 877-881 (2018).

39. Moussa, S. H., Shapiro, A. B., McLeod, S. M. \& A. Miller, A. A. Resistance to sulbactamdurlobactam in clinical isolates of Acinetobacter baumannii is rare and maps to PBP3. Acinetobacter Conference 2019 https://www.entasistx.com/ application/files/3115/6959/0903/Moussa_SUL_ Resistance_Acinetobacter2019_FINAL.pdf. (2019).

40. McLeod, S. M. et al. In vitro antibacterial activity of sulbactam-durlobactam (ETX2514) against 121 recent Acinetobacter baumannii isolates from patients in India. IDweek 2019 https://www.entasistx.com/ application/files/2215/7115/9465/IDweek_2019 ETX2514 India V11 pdf (2019).

41. Barnes, M. D. et al. targeting multidrug-resistant Acinetobacter spp.: sulbactam and the diazabicyclo octenone $\beta$-lactamase inhibitor ETX2514 as a nove therapeutic agent. mBio 10, e00159-19 (2019).

42. Livermore, D. M., Mushtaq, S., Warner, M. \& Woodford, N. Activity of OP0595/ $\beta$-lactam combinations against Gram-negative bacteria with extended-spectrum AmpC and carbapenem-hydrolysing $\beta$-lactamases. J. Antimicrob. Chemother. 70, 3032-3041 (2015)

43. Mushtaq, S., Vickers, A., Woodford, N., Haldimann, A \& Livermore, D. M. Activity of nacubactam (RG6080/ OP0595) combinations against MBL-producing Enterobacteriaceae. J. Antimicrob. Chemother. 74, 953-960 (2018).
44. Okujava, R. et al. Activity of meropenem/nacubactam combination against Gram-negative clinical isolates: ROSCO global surveillance 2017. Open Forum Infect. Dis. 5, S416-S416 (2018).

45. Khan, Z., Iregui, A., Landman, D. \& Quale, J. Activity of cefepime/zidebactam (WCK 5222) against Enterobacteriaceae, Pseudomonas aeruginosa and Acinetobacter baumannii endemic to New York City medical centres. J. Antimicrob. Chemother. $\mathbf{7 4}$, 2938-2942 (2019).

46. Thomson, K. S., AbdelGhani, S., Snyder, J. W. \& Thomson, G. K. Activity of cefepime-zidebactam against multidrug-resistant (MDR) Gram-negative pathogens. Antibiotics 8, 32 (2019).

47. Krajnc, A. et al. Bicyclic boronate VNRX-5133 inhibits metallo- and serine- $\beta$-lactamases. J. Med. Chem. 62 8544-8556 (2019).

48. Liu, B. et al. Discovery of taniborbactam (VNRX-5133): a broad-spectrum serine- and metallo- $\beta$-lactamase inhibitor for carbapenem-resistant bacterial infections J. Med. Chem. https://doi.org/10.1021/acs.jmedchem. 9b01518 (2019).

49. Hackel, M. \& Sahm, D. Antimicrobial activity of cefepime in combination with VNRX-5133 against a collection of $\beta$-lactamase-producing Enterobacteriaceae. ECCMID 2019 https://www.ihma.com/app/uploads/ VenatoRx_P50_FEP-5133-BL_ECCMID-2019_FINAL. pdf (2019)

50. Hackel, M. \& Sahm D. Antimicrobial activity of cefepime in combination with VNRX-5133 against a global 2018 surveillance collection of Pseudomonas aeruginosa. ASM Microbe 2019 https://www.venatorx. com/wp-content/uploads/2019/07/ASMMicrobe2019 SUNDAY-AAR-722.pdf (2019).

51. Castanheira, M., Lindley, J., Huynh, H., Mendes, R. E. $\&$ Lomovskaya, O. Activity of novel $\beta$-lactamase inhibitor QPX7728 combined with $\beta$-lactam agents when tested against carbapenem-resistant Enterobacteriaceae (CRE) isolates. Open. Forum Infect. Dis. 6, S309-S309 (2019).

52. Mendes, R. E., Rhomberg, P. R., Watters, A. A. Castanheira, M., Flamm, R. K. In vitro activity of the orally bioavailable ceftibuten/VNRX-7145 combination against a challenge set of Enterobacteriaceae pathogens carrying molecularly characterized $\beta$-lactamase genes. ECCMID 19 https://www.jmilabs. com/data/posters/ECCMID 19-VNRX-7145-ceftibuten. pdf (2019).

53. John, K. J., Chatwin, C. L., Hamrick, J. C., Moeck, C. $\&$ Pevear, D. C. Rescue of ceftibuten activity by the oral $\beta$-lactamase inhibitor VNRX-7145 against Enterobacteriaceae expressing class A, C and/or D $\beta$-lactamases. ASM Microbe $2019 \mathrm{https}$ ://www. venatorx.com/wp-content/uploads/2019/07/ ASMMicrobe2019-SUNDAY-AAR-719.pdf (2019).

54. M. Hackel $\&$ Sahm, D. In vitro activity of ceftibuten in combination with VNRX-7145 and comparators against 1,066 UTI isolates non-susceptible to amoxicillin-clavulanate and levofloxacin microbe. ASM Microbe 2019 https://www.venatorx.com/ wp-content/uploads/2019/07/ASMMicrobe2019 SUNDAY-AAR-721.pdf (2019).

55. McLeod, S et al. The antibacterial activity of sulbactam and the novel b-lactamase Inhibitor ETX2514 combined with imipenem or meropenem against recent clinical isolates of Acinetobacter baumannii and Pseudomonas aeruginosa. ASM Microbe 2017 https://www.entasistx.com/application files/2815/1846/7310/McLeod-et-al-ASM-Microbe2017-FRI-82.pdf (2017)

56. Duncan, L. R., Rhomberg, P. R., Mendes, R. E. Flamm, R. K. \& Trias, J. Ceftibuten-avibactam activity against $\beta$-lactam-resistant Enterobacteriaceae clinical isolates. ASM Microbe 2019 https://www.jmilabs.com/ data/posters/ASM-Microbe 19-ceftibuten-avibactam. pdf (2019).

57. Papp-Wallace, K. M. et al. Beyond piperacillintazobactam: cefepime and AAl101 as a potent $\beta$-Lactam- $\beta$-lactamase inhibitor combination. Antimicrob. Agents Chemother. 63, e00105-e00119 (2019).

58. Gaibani, P. et al. In vivo evolution of resistant subpopulations of KPC-producing Klebsiella pneumoniae during ceftazidime/avibactam treatment. J. Antimicrob. Chemother. 73, 1525-1529 (2018).

59. Bush, K. Past and present perspectives on $\beta$-lactamases. Antimicrob. Agents Chemother. 62 e01076-18 (2018) This study presents an extensive overview of $\beta$-lactamases including evolution and present situation. 
60. Veeraraghavan, B. et al. Newer $\beta$-lactam $/ \beta$-lactamase inhibitor for multidrug-resistant gram-negative infections: challenges, implications and surveillance strategy for India. Indian J. Med. Microbiol. 36 334-343 (2018)

61. Negash, K. H., Norris, J. K. S. \& Hodgkinson, J. T. Siderophore-antibiotic conjugate design: new drugs for bad bugs? Molecules 24, 3314 (2019).

62. Ito, A. et al. Siderophore cephalosporin cefiderocol utilizes ferric iron transporter systems for antibacterial activity against $P$ seudomonas aeruginosa. Antimicrob. Agents Chemother. 60, 7396-7401 (2016).

63. Jacobs, M. R. et al. ARGONAUT-I: Activity of cefiderocol (S-649266), a siderophore cephalosporin, against gram-negative bacteria, including carbapenemresistant nonfermenters and Enterobacteriaceae with defined extended-spectrum $\beta$-lactamases and carbapenemases. Antimicrob. Agents Chemother. 63, e01801-e01818 (2018)

64. Karlowsky, J. A. et al. In vitro activity of cefiderocol, a siderophore cephalosporin, against gram-negative bacilli isolated by clinical laboratories in North America and Europe in 2015-2016: SIDERO-WT-2015. Int. J. Antimicrob. Agents 53, 456-466 (2019).

65. Kazmierczak, K. M. et al. In vitro activity of cefiderocol, a siderophore cephalosporin, against a recent collection of clinically relevant carbapenem-non-susceptible Gram-negative bacilli, including serine carbapenemaseand metallo- $\beta$-lactamase-producing isolates (SIDEROWT-2014 study). Int. J. Antimicrob. Agents 53 177-184 (2019).

66. Echols, R., Ariyasu, M. \& Nagata, T. D. Pathogenfocused clinical development to address unmet medical need: cefiderocol targeting carbapenem resistance. Clin. Infect. Dis. 69, S559-S564 (2019).

67. Food and Drug Administration. FDA briefing document: meeting of the Antimicrobial Drugs Advisory Committee (AMDAC) https://www.fda.gov/ media/131703/download (2019)

68. Blais, J. et al. In vitro activity of LYS228, a nove monobactam antibiotic, against multidrug-resistant Enterobacteriaceae. Antimicrob. Agents Chemother. 62, e00552-18 (2018)

69. Dean, C. R. et al. Mode of action of the monobactam LYS228 and mechanisms decreasing in vitro susceptibility in escherichia coli and Klebsiella pneumoniae. Antimicrob. Agents Chemother. 62, e01200-e01218 (2018)

70. Dunne, M. Huband, M., Flamm, R., Aronin, S. \& Puttagunta, S. Prediction of sulopenem activity against Enterobacteriaceae using ertapenem as a surrogate. ASM Microbe $2018 \mathrm{https}$ ://d1 io3yog0oux5.cloudfront. net/_1af31c1063e9451129263f05d42bc5fa/iterumtx db/395/2664/pdf/ASM+2018_Ertapenem+surrogate. Final.pdf (2018)

71. Iterum Therapeutics. Press release 10. 12. 2019 Iterum Therapeutics announces topline results from phase III clinical trial of oral and IV sulopenem for the treatment of complicated intra-abdominal infections. https://www.globenewswire.com/newsrelease/2019/12/10/1958907/0/en/IterumTherapeutics-Announces-Topline-Results-from-Phase3-Clinical-Trial-of-Oral-and-IV-Sulopenem-for-theTreatment-of-Complicated-Intra-abdominal-Infections html (2019).

72. Karlowsky, J. A. et al. In vitro activity of sulopenem, an oral penem, against urinary isolates of escherichia coli. Antimicrob. Agents Chemother. 63, e01832-18 (2018).

73. McEntee, L. et al. Pharmacodynamics of tebipenem: new options for oral treatment of multidrug-resistant Gram-negative infections. Antimicrob. Agents Chemother. 63, e00603-e00619 (2019).

74. Richter, S. E. et al. Risk factors for development of carbapenem resistance among gram-negative rods. Open Forum Infect. Dis. 6, ofz027 (2019).

75. Thaker, M., Spanogiannopoulos, P. \& Wright, G. D. The tetracycline resistome. Cell Mol. Life Sci. 67, 419-431 (2010)

This study presents an overview of structure, mechanism and regulation of the genes and proteins associated with tetracycline resistance.

76. Villa, L., Feudi, C., Fortini, D., García-Fernández, A \& Carattoli, A. Genomics of KPC-producing Klebsiella pneumoniae sequence type 512 clone highlights the role of RamR and ribosomal S10 protein mutations in conferring tigecycline resistance. Antimicrob. Agents Chemother. 58, 1707-1712 (2014)

77. Park, J. et al. Plasticity, dynamics, and inhibition of emerging tetracycline resistance enzymes. Nat. Chem. Biol. 13, 730-736 (2017).
78. Sun, J. et al. Plasmid-encoded tet $(X)$ genes that confer high-level tigecycline resistance in Escherichia coli. Nat. Microbiol. 4, 1457-1464 (2019).

79. Grossman, T. H. Tetracycline antibiotics and resistance. Cold Spring Harb. Perspect. Med. 6 , a025387-a025387 (2016).

80. He, T. et al. Emergence of plasmid-mediated high-level tigecycline resistance genes in animals and humans. Nat. Microbiol. 4, 1450-1456 (2019).

81. Zhang, Y., Lin, X. \& Bush, K. In vitro susceptibility of $\beta$-lactamase-producing carbapenem-resistant Enterobacteriaceae (CRE) to eravacycline. J. Antibiotics 69, 600-604 (2016).

82. Pfaller, M. A., Rhomberg, P. R., Huband, M. D. \& Flamm, R. K. Activity of omadacycline tested against Enterobacteriaceae causing urinary tract infections from a global surveillance program (2014). Diagn. Microbiol. Infect. Dis. 91, 179-183 (2018).

83. Kaminishi, T. et al. Third-generation tetracycline KBP-7072 exploit reveal a new potential primary tetracycline binding pocket. Preprint at https://doi.org 10.1101/508218 (2018).

84. Yang, F., Wang, Y., Wang, P., Hong, M. \& Benn, V. Multiple ascending dose safety, tolerability, and pharmacokinetics of KBP-7072, a novel third-generation tetracycline. Open For. Infect. Dis. 4, S291-S291 (2017).

85. Huband, M. D. et al. Activity of KBP-7072 against recent and molecularly characterized Acinetobacter baumannii isolates. ASM/ESCMID Drug Development Conference 2019 https://www.jmilabs.com/data/ posters/ASM-ESCMID2019-KBP-7072-Acinetobacter. pdf (JMI Labs, 2019).

86. Falagas, M. E. et al. Activity of TP-6076 against carbapenem-resistant Acinetobacter baumannii isolates collected from inpatients in Greek hospitals. Int. J. Antimicrob. Agents 52, 269-271 (2018).

87. Fyfe, C., LeBlanc, G., Close, B. \& Newman J. TP-6076 is active against carbapenem- and polymyxin-resistant Enterobacteriaceae and Acinetobacter baumanni isolates. ECCMID 2017 https://www.escmid.org/ typo3conf/ext/escmid_solr/Resources/Images/icn abstract-hover.png (Vienna, 2017).

88. Sun, C. et al. TP-6076, a fully synthetic tetracycline antibacterial agent, is highly potent against a broad range of pathogens, including carbapenem-resistant Enterobacteriaceae. ASM Microbe 2017 https://www. tphase.com/wp-content/uploads/2019/04/Sun-S ASM_2017_SUN-332-poster_6076-SAR_FINAL.pdf (2017).

89. Tsai, L. \& Moore, A. Safety, tolerability, and pharmacokinetics of multiple doses of TP-6076, a novel, fully synthetic tetracycline, in a phase I study. Open Forum Infect. Dis. 5, S420-S420 (2018).

90. Grossman, T. H. et al. Fluorocycline TP-271 is potent against complicated community-acquired bacterial pneumonia pathogens. mSphere 2, e00004-e00017 (2017).

91. Aruhomukama, D., Sserwadda, I. \& Mboowa, G. Investigating colistin drug resistance: the role of high-throughput sequencing and bioinformatics. F100OResearch 8, 150-150 (2019).

92. Brown, P. et al. Design of next generation polymyxins with lower toxicity: the discovery of SPR206. ACS Infect. Dis. 5, 1645-1656 (2019).

93. Corbett, D. et al. Potentiation of antibiotic activity by a novel cationic peptide: potency and spectrum of activity of SPR741. Antimicrob. Agents Chemother. 61, e00200-e00217 (2017).

94. Fleischmann, W. A., Greenwood-Quaintance, K. E. \& Patel, R. In vitro activity of plazomicin compared to amikacin, gentamicin, and tobramycin against multidrug resistant aerobic Gram-negative bacilli. Antimicrob. Agents Chemother. https://doi.org/ 10.1128/AAC.01711-19 (2019).

95. Serio, A., Keepers, T., Andrews, L. \& Krause, K Aminoglycoside revival: review of a historically important class of antimicrobials undergoing rejuvenation. EcoSal Plus https://doi.org/10.1128/ ecosalplus.ESP-0002-2018 (2018)

96. Livermore, D. M. et al. Activity of aminoglycosides, including ACHN-490, against carbapenem-resistant Enterobacteriaceae isolates. J. Antimicrob. Chemother 66, 48-53 (2010)

97. Juhas, M. et al. In vitro activity of apramycin against multidrug-, carbapenem- and aminoglycoside-resistan Enterobacteriaceae and Acinetobacter baumannil. J. Antimicrob. Chemother. 74, 944-952 (2019).

98. Hunter, J. E., Hart, C. A., Shelley, J. C., Walton, J. R. $\&$ Bennett, M. Human isolates of apramycin-resistant Escherichia coli which contain the genes for the
AAC(3)IV enzyme. Epidemiol. Infect. 110, 253-259 (1993).

99. Yang Y, Hu, F \& Zhu, D. evaluation of contezolid activity to anaerobic and gram-positive-cocci isolates from a phase III acute bacterial skin and skin structure infection clinical trial (MRX-I-06). Open Forum Infect. Dis. 6, S315-S315 (2019).

100. Flamm, R. K., Rhomberg, P. R. \& Sader, H. S. In vitro activity of the novel lactone ketolide nafithromycin (WCK 4873) against contemporary clinical bacteria from a global surveillance program. Antimicrob. Agents Chemother. 61, e01230-17 (2017).

101. Fisher, C. et al. Activity of TNP-2092 against biofilms formed by prosthetic joint infection-associated staphylococci. Open Forum Infect. Dis. 6, S313-S313 (2019).

102. Yao, J., Maxwell, J. B. \& Rock, C. O Resistance to AFN-1252 arises from missense mutations in Staphylococcus aureus enoyl-acyl carrier protein reductase (Fabl). J. Biol. Chem. 288, 36261-36271 (2013).

103. Schiebel, J. et al. Staphylococcus aureus Fabl: inhibition, substrate recognition, and potential implications for in vivo essentiality. Structure $\mathbf{2 0}$ 802-813 (2012)

104. Hawser, S. et al. Activity of debio 1452 against Staphylococcus spp. collected in 2013/2014. ECCMID 2016 https://www.ihma.com/app/uploads/ P139_POSTER_Debiopharm_IHMA_ECCMID_2016 v1-final.pdf (2016).

105. Menetrey, A. et al. Bone and joint tissue penetration of the Staphylococcus-selective antibiotic afabicin in patients undergoing elective hip replacement surgery. Antimicrob. Agents Chemother. 63, e01669-18 (2019).

106. Escaich, S. et al. The MUT056399 Inhibitor of Fabl is a new antistaphylococcal compound. Antimicrob. Agents Chemother. 55, 4692-4697 (2011).

107. Kaul, M. et al. TXA709, an FtsZ-targeting benzamide prodrug with improved pharmacokinetics and enhanced in vivo efficacy against methicillin-resistant Staphylococcus aureus. Antimicrob. Agents Chemother. 59, 4845 (2015)

108. Kaul, M., Mark, L., Parhi, A. K., LaVoie, E. J. \& Pilch, D. S. Combining the FtsZ-targeting prodrug TXA709 and the cephalosporin cefdinir confers synergy and reduces the frequency of resistance in methicillin-resistant Staphylococcus aureus. Antimicrob. Agents Chemother. 60, 4290 (2016).

109. Basarab, G. S. et al. Responding to the challenge of untreatable gonorrhea: ETX0914, a first-in-class agent with a distinct mechanism-of-action against bacterial type II topoisomerases. Sci. Rep. 5, 11827-11827 (2015).

110. Taylor, S. N. et al. Gepotidacin for the treatment of uncomplicated urogenital gonorrhea: a phase II, randomized, dose-ranging, single-oral dose evaluation. Clin. Infect. Dis. 67, 504-512 (2018)

111. Negash, K. et al. The metabolism and disposition of GSK21 40944 in healthy human subjects. Xenobiotica 46, 683-702 (2016)

112. Flamm, R. K., Farrell, D. J., Rhomberg, P. R. Scangarella-Oman, N. E. \& Sader, H. S. Gepotidacin (CSK2140944) in vitro activity against Gram-positive and Gram-negative bacteria. Antimicrob. Agents Chemother. 61, e00468-17 (2017)

113. Bisacchi, G. S. \& Manchester, J. I. A new-class antibacterial - almost. Lessons in drug discovery and development: a critical analysis of more than 50 years of effort toward ATPase inhibitors DNA gyrase topoisomerase IV. ACS Infect. Dis. 1, 4-41 (2015).

This study presents insights into the historical attempts to discover and develop ATPase inhibitors of gyrase and topo IV as new-class antibacterial agents with broad-spectrum potential.

114. Brown-Elliott, B. A., Rubio, A. \& Wallace, R. J. Jr. In vitro susceptibility testing of a novel benzimidazole, SPR719, against nontuberculous mycobacteria. Antimicrob. Agents Chemother. 62, e01503-e01518 (2018).

115. Food \& Drug Administration. Drug approval package: XENLENTA https://www.accessdata.fda.gov/ drugsatfda docs/nda/2019/2116720rig 1 s000, 2116730rig 1s000TOC.cfm (2020).

116. Cho, J. C., Crotty, M. P. \& Pardo, J. Ridinilazole: a novel antimicrobial for Clostridium difficile infection. Ann. Gastroenterol. 32, 134-140 (2019).

117. Bassères, E. et al. Understanding the Mechanism of Action of Ridinilazole (SMT19969), a Novel Treatment for Clostridium difficile. ECCMID 2016 https://www.escmid.org/escmid_publications/escmid_ elibrary/material/? mid=50079 (2016). 
118. Khalaf, A. I. et al. Distamycin analogues with enhanced lipophilicity: synthesis and antimicrobial activity. J. Med. Chem. 47, 2133-2156 (2004).

119. Garey, K. W. et al. A Randomized, blinded, placebo- and vancomycin-controlled, first-in-human (FIH) study of the safety, pharmacokinetics (PK), and fecal microbiome effects of ACX-362E, a novel anti-clostridial DNA polymerase IIIC (pollIIC) inhibitor. Open Forum Infect. Dis. 6, S995-S996 (2019).

120. Green, L. S. et al. Inhibition of methionyl-tRNA synthetase by REP8839 and effects of resistance mutations on enzyme activity. Antimicrob. Agents Chemother. 53, 86-94 (2009).

121. Roberts, J. A. et al. Individualised antibiotic dosing for patients who are critically ill: challenges and potential solutions. Lancet Infect. Dis. 14, 498-509 (2014).

122. Livermore, D. M. The 2018 Garrod Lecture: preparing for the black swans of resistance. J. Antimicrob. Chemother. 73, 2907-2915 (2018).

123. Amaral, L., Martins, A., Spengler, G. \& Molnar, J. Efflux pumps of Gram-negative bacteria: what they do, how they do it, with what and how to deal with them. Front. Pharmacol. 4, 168 (2014).
Acknowledgements

The authors thank S. Paulin and P. Beyer (WHO) and the members of the advisory group of the WHO pipeline report - M. Butler, L. Czaplewski, J. Hood, F. Franceschi, R. Kozlov, C. Lienhardt, N. Ohmagari, L. Silver and R. Alm - for their support, advice and contributions.

\section{Author contributions}

U.T. wrote the article. U.T., K.B., S.H., M.P., J.H.R., E.T. and G.E.T reviewed and edited the manuscript before submission.

\section{Competing interests}

U.T., M.P., E.T., and G.E.T. declare no financial relationships with any organizations that might have an interest in the sub mitted work. K.B. receives retirement compensation from Bristol-Myers Squibb, Johnson \& Johnson and Pfizer, and is a shareholder in Entasis, Fedora and Johnson \& Johnson. In the past year, she has served as a consultant or scientific advisory board member for Allecra, Entasis, Fedora, Forma Gladius, Mutabilis and VenatoRx. J.H.R. reports holding positions as Chief Medical Officer \& Director at F2G Non-Executive Director and Consultant at Adenium Biotech, Operating Partner and Consultant at Advent Life Sciences and Expert-in-Residence at the Wellcome Trust, as well as being a member of the scientific advisory boards of
Macrolide Pharmaceuticals, Bugworks Research, Basilea Pharmaceutica, Forge Therapeutics and Novo Holdings. He reports personal fees from Phico Therapeutics, ABAC Therapeutics, Polyphor, Heptares Therapeutics, Gangagen, Meiji Seika Pharma, Basilea Pharmaceutica International, Allecra Therapeutics, Forge Therapeutics, SinSa Labs, AtoxBio, Peptilogics, F. Hoffmann-LaRoche and Novo Holdings; he is a shareholder in AstraZeneca, F2G, Adenium Biotech, Advent Life Sciences, Macrolide Pharmaceuticals and Bugworks Research. S.H. reports receiving grants from IMI Brussels during the conduct of the present study, as well as grants from Pfizer, personal fees from Novartis, personal fees from DNA Electronics, personal fees from Bayer and personal fees from GSK outside the submitted work.

\section{Publisher's note}

Springer Nature remains neutral with regard to jurisdictional claims in published maps and institutional affiliations.

\section{RELATED LINKS}

WHO clinical pipeline report: https://apps.who.int/iris/

bitstream/handle/10665/330420/9789240000193-eng.pdf

(C) Springer Nature Limited 2020 